\title{
Uso de modelos paramétricos para estimativas de investimento aplicadas às etapas de britagem primária e secundária de minério de ferro
}

\author{
Paulo César Salvador de Aguiar Júnior ${ }^{*} *$ (1) \\ Douglas Batista Mazzinghy ${ }^{l}$ \\ Carlos Esteves Teixeira ${ }^{I}$ \\ Fábio Paival
}

\section{Resumo}

Estimativas de custos e investimentos assertivas elevam a confiabilidade da avaliação econômica e financeira dos projetos, avaliação esta que tem a finalidade de validar ou não o prosseguimento de estudos e projetos do setor de mineração. As etapas de britagem primária e secundária na mineração podem ser consideradas como as etapas iniciais do beneficiamento de minérios, usualmente aplicadas após as etapas de desmonte, carregamento e transporte. Para determinação dos modelos paramétricos estimadores este artigo propõe modelos de regressão multivariados utilizando a metodologia sugerida por Sayadi et al. [1]. Esses modelos foram desenvolvidos usando uma técnica de regressão multivariada (MVR) baseada na análise de componentes principais (PCA). O resultado da avaliação dos modelos mostrou que as taxas médias de erro relativo foram de $17,7 \%$ utilizando dois componentes principais e chegaram a $9,7 \%$ quando foram mantidos os componentes principais originais. Em análise complementar foram ajustadas as equações propostas por O'Hara em 1980 [2] e 1992 [3], buscando avaliar se essas equações podem refletir a realidade de custos e investimentos de mineração no Brasil em 2019. Os erros médios encontrados utilizando os fatores de ajuste propostos foram de $17 \%$ para as equações atualizadas de 1992 e 20\% para as equações originais de 1980 [2], mostrando assim que estes modelos ainda possuem boa aplicabilidade para estudos de viabilidade.

Palavras-chave: Britagem; Estimativa de custos; Regressão; Análise de componentes principais; Programação Linear.

\section{Use of parametric models for investment estimates applied to the primary and secondary crushing stages of iron ore}

\begin{abstract}
Assertive cost and investment estimates raise the reliability of the economic and financial evaluation of projects. Primary and secondary crushing stages in mining can be considered as the initial stages of mineral processing, usually after the stages of blasting, loading and hauling. In order to determine the parametric estimator models, this paper proposes multivariate regression models using the methodology suggested by Sayadi et al. [1]. These models were developed using a multivariate regression technique based on principal component analysis (PCA). The results of the evaluation of the models showed that the mean relative error rates were $17.7 \%$ using two main components and reached $9.7 \%$ when the original main components were maintained (lower than the multivariate regression using the stepwise method with error of 10.9\%). In complementary analysis were adjusted the equations proposed by O'Hara in 1980 [2] and 1992 [3], seeking to evaluate if these equations can reflect the reality of mining investments in Brazil in 2019. The average errors found using the adjustment factors proposed with the optimization tool were $17 \%$ for the updated equations of 1992 and $20 \%$ for the original equations of 1980 [2] showing that these models still have good applicability for feasibility studies.
\end{abstract}

Keywords: Crushing; Cost estimation; Regression; Principal component analysis; Linear Programming.

\footnotetext{
${ }^{I}$ Departamento de Engenharia de Minas, Universidade Federal de Minas Gerais - UFMG, Belo Horizonte, MG, Brasil.

*Autor correspondente: pcsajpc@yahoo.com.br
} 


\section{Introdução}

O grande desafio de uma estimativa de investimento ocorre quando não existe uma grande gama de informações relevantes disponíveis, o que reforça a necessidade da construção de modelos paramétricos confiáveis. Modelos multivariáveis tendem a retratar melhor os modelos de custos e investimentos das operações unitárias de mineração, uma vez que o conceito das instalações depende de variáveis correlatas entre si, como a característica do minério, a configuração de arranjo proposta pela engenharia de projetos, as especificidades do local de implantação, tipo de terreno, topografia, etc. Segundo Sayadi et al. [1], historicamente diversos modelos de estimativa de custos foram desenvolvidos. Pesquisadores incluindo O'Hara [2], O'Hara e Suboleski [3], Prasad [4], Mular [5] e outros tentaram fornecer modelos de estimativa de custos e investimentos apropriados em mineração. Quase todos eles empregaram abordagens de regressão exponencial univariada, apenas correlacionando uma variável chave ao valor de custo. As etapas de britagem primária e secundária têm relevante papel na cominuição do Run of Mine (RoM) e, no caso especificamente da britagem secundária, também tem importante papel de proteção do sistema de transportadores de correias posteriores ao circuito de britagem secundária (em muitos casos de longa distância, neste caso quanto maior a quantidade de transferências, maior o risco de rasgo). O rasgo de correias ocorre devido à presença de materiais lamelares que passam diretamente pelo britador primário.

A utilização de grelhas vibratórias de escalpe antes do britador primário também é útil no sentido de reduzir a massa alimentada ao britador primário, reduzindo em alguns casos seu porte, e também por criar um "colchão de finos" no transportador denominado como "transportador de sacrifício", que recebe o material britado e assim reduz o risco de rasgo de correias. Aspectos relativos ao grau de complexidade locacional, topográfica, de infraestrutura (balanço corte/aterro), dos tipos de contenção (muro de terra armada, geogrelha, tirantes, e outros) e fundações aplicados; também são relevantes quando se trata da análise dos valores de investimentos de britagens primárias e secundárias, mas não serão foco deste estudo. Neste trabalho funções de regressão multivariada foram fornecidas a partir de uma base de dados atualizada, para a estimativa dos investimentos de instalações fixas de britagem primária e secundária. Além disso, foi feita a comparação dos dados reais disponibilizados de britagens implantadas e projetadas no Brasil recentemente com os modelos paramétricos propostos por O'Hara em 1980 [2] e 1992 [3], corrigidos por fatores de ajuste geradores dos erros médios mínimos.

\section{Metodologia}

\subsection{Classificação dos circuitos}

Um conjunto de dados técnicos e econômicos para oito tipos de circuitos de britagens primárias e secundárias foi coletado. Os circuitos de britagem escolhidos envolvem como equipamentos principais britadores de mandíbulas ou giratórios (britadores primários), britadores cônicos (britadores secundários), grelhas vibratórias de escalpe (para classificação anterior à alimentação do britador primário), peneiras vibratórias inclinadas (normalmente do tipo banana, para classificação anterior à alimentação do britador secundário), alimentadores de sapatas, alimentadores de correias ou vibratórios, e transportadores de correia convencionais. Estes circuitos foram escolhidos por serem os circuitos mais comuns nas operações e projetos de minério de ferro no Brasil.

A Figura 1 mostra os fluxogramas de processo dos circuitos de britagem analisados. Os dados de investimentos dos projetos foram classificados conforme os circuitos mostrados na Figura 1, atualizados em sua data-base, ajustados para uma base cambial comum de 3,50 (R\$/U\$) e normalizados garantindo a confidencialidade dos valores de investimentos. Estes dados são apresentados na Tabela 1.

Os circuitos denominados C, D, E, G e H utilizam britadores primários giratórios, aplicados quando existe no RoM participação relevante de itabiritos compactos (minério em que a amostra britada com top size de $50 \mathrm{~mm}$ possui retido superior a 50\% na malha de $8,0 \mathrm{~mm}$ ). Para os demais tipos de itabiritos presentes no quadrilátero ferrífero (principalmente os friáveis) e hematitas, comumente utiliza-se como britadores primários os britadores do tipo mandíbulas. Todas as britagens analisadas são fixas ou semi-móveis (moduladas de forma a facilitar futura desmontagem e relocação). Os investimentos associados aos circuitos de britagem utilizados no estudo se referem aos custos diretos de implantação, que consideram toda a parte de fornecimentos de equipamentos, materiais e serviços de montagem e obras civis, incluindo instalações provisórias (canteiros de obras). Valores de investimentos indiretos, como contingências, compensações ambientais, gerenciamento de obra por parte do contratante, engenharia detalhada, despesas pré-operacionais e owner team estão fora do escopo.

A Tabela 1 apresenta os dados das britagens analisadas.

\subsection{Regressão multivariada}

A metodologia de modelagem utilizada seguiu a metodologia proposta por Sayadi et al. [1], conforme apresentado na Figura 2.

A medida de erro foi usada como um meio de comparação entre os modelos. O erro médio percentual foi definido como apresentado na Equação 1 [6]:

$$
\text { Erro }=\frac{\sum\left|\left(\frac{\mathrm{C}_{\mathrm{e}}-\mathrm{C}_{\mathrm{a}}}{\mathrm{C}_{\mathrm{a}}}\right) \times 100\right|}{n}
$$

Onde $C_{e}$ é o valor estimado das britagens, $C_{a}$ é o valor real (base de dados) e $n$ é o número de informações utilizadas 

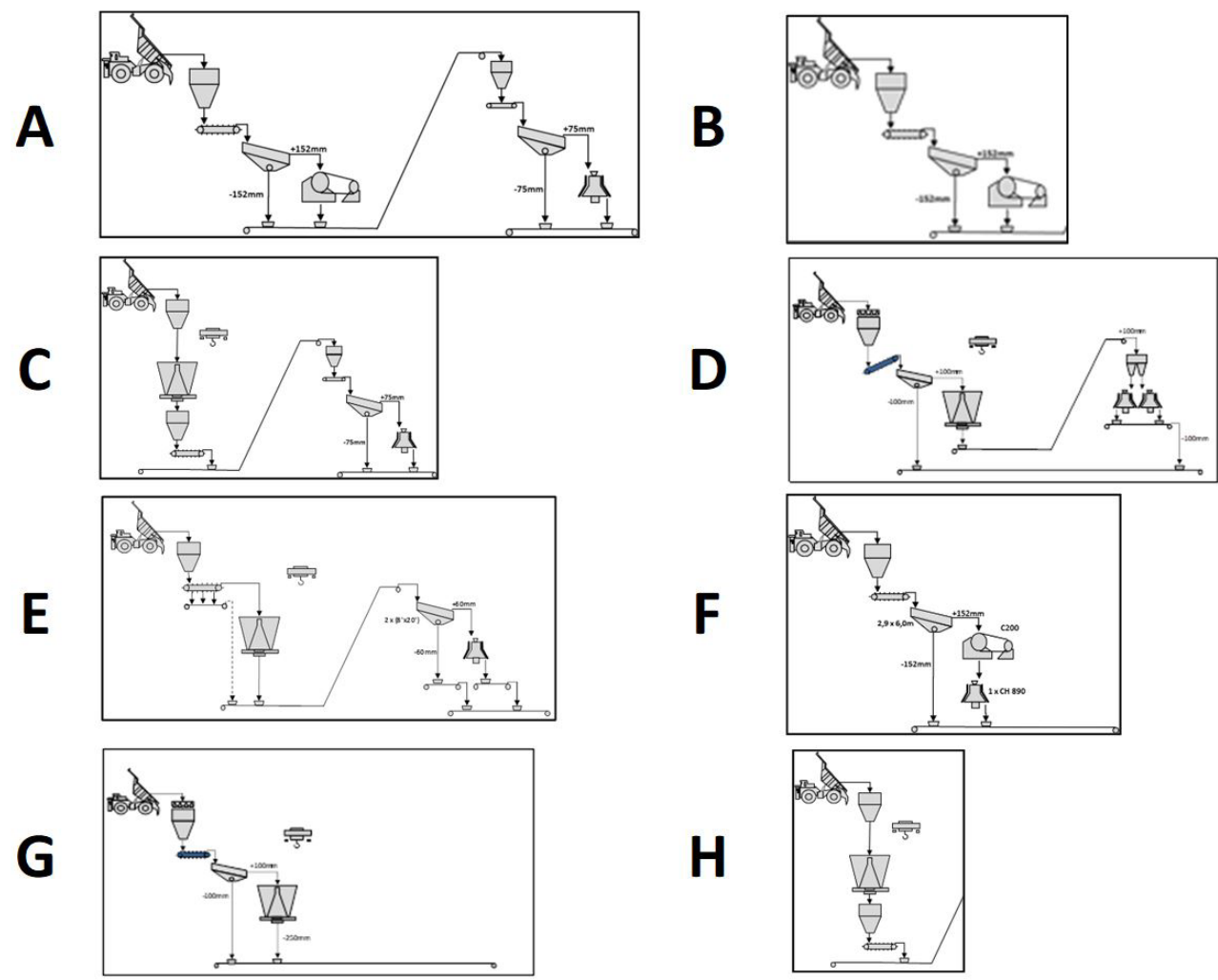

Figura 1. Circuitos típicos de britagem primária e secundária.

Tabela 1. Dados técnicos e econômicos das britagens

\begin{tabular}{|c|c|c|c|c|c|c|c|c|c|c|}
\hline Item & Projeto & $\begin{array}{l}\text { Tipo de } \\
\text { Circuito }\end{array}$ & $\begin{array}{c}\text { Caminhão } \\
\text { (t) } \\
{[\mathrm{T}]}\end{array}$ & $\begin{array}{c}\text { Produção } \\
\text { (t/h base } \\
\text { úmida) } \\
{[C]}\end{array}$ & $\begin{array}{c}\text { Top size } \\
\text { (mm) } \\
{[\mathrm{S}]}\end{array}$ & $\begin{array}{c}\% \text { da } \\
\text { aliment. } \\
\text { retido em } \\
100 \mathrm{~mm} \\
{[\mathrm{G}]} \\
\end{array}$ & $\begin{array}{c}\text { \% limite de } \\
\text { compacto } \\
\text { na } \\
\text { alimentação } \\
{[\mathrm{K}]} \\
\end{array}$ & $\begin{array}{c}\text { Altura do } \\
\text { prédio (m) } \\
{[\mathrm{H}]}\end{array}$ & $\begin{array}{c}\text { Volume } \\
\text { útil moega } \\
\text { alimentação } \\
\left(\mathbf{m}^{3}\right)[\mathbf{V}]\end{array}$ & $\begin{array}{c}\text { Investimento } \\
\$ \\
\text { (base 100) }\end{array}$ \\
\hline 1 & $\eta$ & A & 190 & 4.131 & 1.000 & 24,7 & 75 & 22,0 & 190 & 1,00 \\
\hline 2 & $\eta$ & A & 190 & 4.131 & 1.000 & 24,7 & 75 & 22,0 & 190 & 1,01 \\
\hline 3 & $\sigma$ & B & 329 & 4.000 & 1000 & 13,5 & 30 & 26,0 & 670 & 1,09 \\
\hline 4 & $\delta$ & B & 329 & 4.000 & 1000 & 13,5 & 30 & 30,0 & 670 & 1,15 \\
\hline 5 & $\gamma$ & B & 329 & 8.000 & 1200 & 15,0 & 10 & 24,8 & 655 & 1,19 \\
\hline 6 & $v$ & B & 240 & 4.636 & 960 & 10,0 & 5 & 22 & 247 & 0,76 \\
\hline 7 & $\phi$ & $\mathrm{C}$ & 240 & 4.735 & 1200 & 85,0 & 100 & 28,0 & 305 & 1,02 \\
\hline 8 & $\eta$ & $\mathrm{C}$ & 190 & 4.131 & 1.000 & 24,7 & 75 & 26,2 & 190 & 1,02 \\
\hline 9 & $\eta$ & $\mathrm{C}$ & 190 & 4.131 & 1.000 & 24,7 & 75 & 26,2 & 190 & 1,01 \\
\hline 10 & $\alpha$ & D & 292 & 7.272 & 1200 & 20,0 & 22 & 37,6 & 800 & 1,96 \\
\hline 11 & $\alpha$ & D & 292 & 7.272 & 1200 & 20,0 & 22 & 43,0 & 800 & 1,91 \\
\hline 12 & $\alpha$ & D & 292 & 7.272 & 1200 & 20,0 & 22 & 39,0 & 800 & 1,89 \\
\hline 13 & $\eta$ & $\mathrm{E}$ & 190 & 4.131 & 1.000 & 24,7 & 75 & 24,0 & 190 & 1,07 \\
\hline 14 & $\eta$ & E & 190 & 4.131 & 1.000 & 24,7 & 75 & 24,0 & 190 & 1,06 \\
\hline 15 & $\eta$ & $\mathrm{F}$ & 190 & 4.131 & 1.000 & 24,7 & 75 & 25,0 & 190 & 0,92 \\
\hline 16 & $\pi$ & $\mathrm{F}$ & 240 & 4.145 & 960 & 10,0 & 5 & 27,8 & 247 & 0,96 \\
\hline 17 & v & $\mathrm{F}$ & 240 & 4.636 & 960 & 10,0 & 5 & 28,0 & 247 & 0,63 \\
\hline 18 & $v$ & $\mathrm{~F}$ & 240 & 4.636 & 960 & 10,0 & 10 & 31,8 & 400 & 1,01 \\
\hline 19 & $\omega$ & $\mathrm{F}$ & 130 & 2.336 & 960 & 15,6 & 0 & 25,0 & 180 & 0,42 \\
\hline 20 & $\alpha$ & G & 292 & 7.272 & 1200 & 20,0 & 22 & 38,5 & 800 & 1,69 \\
\hline 21 & $\alpha$ & G & 292 & 7.272 & 1200 & 20,0 & 22 & 33,1 & 800 & 1,59 \\
\hline 22 & $\mu$ & $\mathrm{G}$ & 329 & 8.000 & 1200 & 15 & 10 & 30,9 & 643 & 2,28 \\
\hline
\end{tabular}




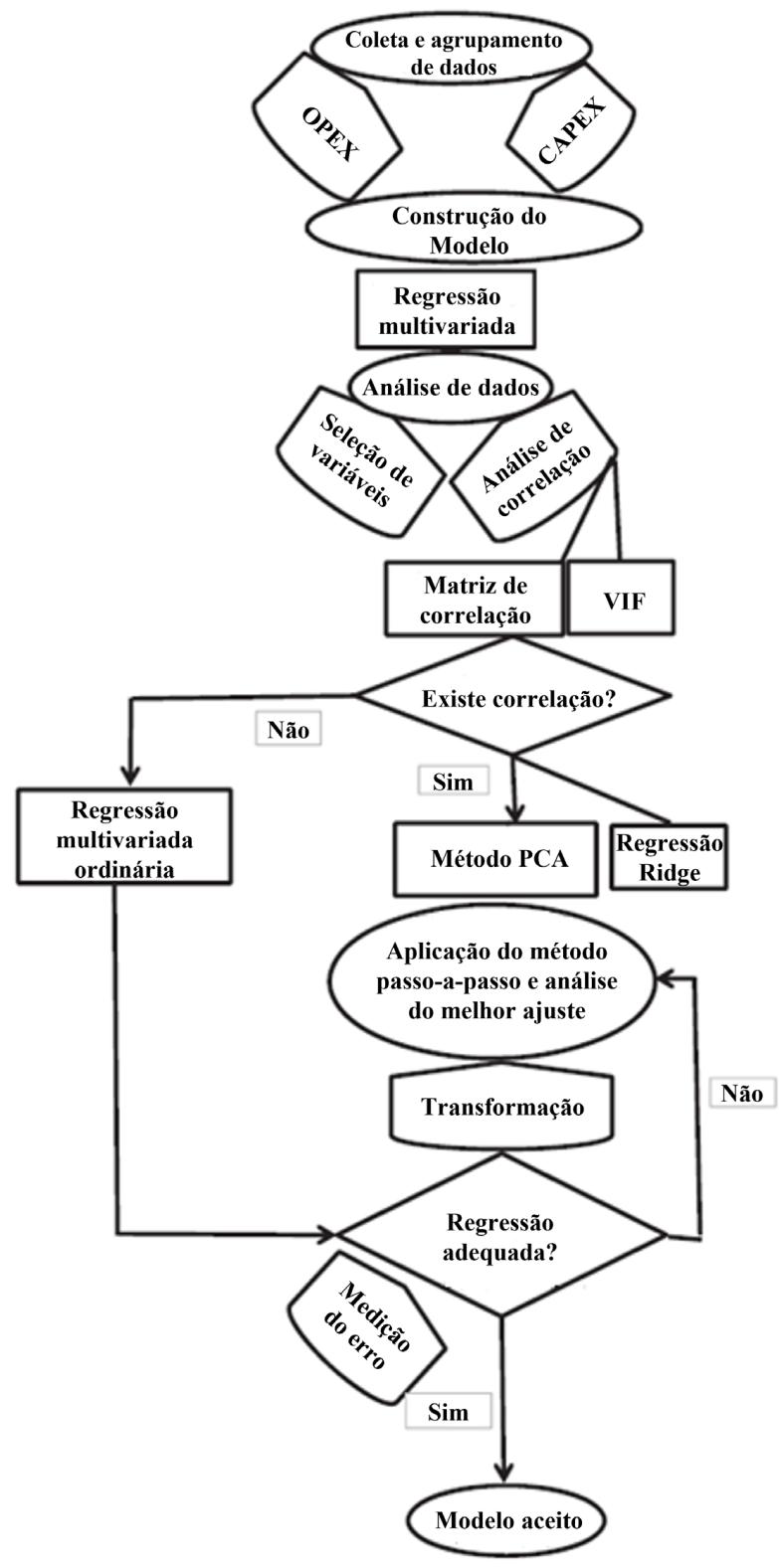

Figura 2. Esquemático da metodologia utilizada [1], modificado.

para a construção do modelo de regressão. Como nesta pesquisa encontrou-se correlação significativa entre as variáveis na construção dos modelos, a solução proposta para a resolução de multicolinearidade foi a aplicação do método de Análise de Componentes Principais (PCA, Principal Component Analysis), a fim de eliminar as dependências entre as variáveis. PCA é um dos métodos multivariados mais simples e é muito utilizado quando variáveis são originárias de um processo em que diversas características devem ser observadas ao mesmo tempo, explicando a estrutura de variância e covariância de um vetor aleatório, através da construção de combinações lineares das variáveis originais do processo. Ou seja, se trata de uma técnica de redução da dimensão dos dados que visa encontrar combinações lineares (denominados componentes principais, PC's) das variáveis que retêm a maior parte da variância da amostra. Para fazer isso, o método maximiza individualmente a variância de cada componente principal, mantendo-os não correlacionados entre si. A técnica permite revelar relações que não eram previamente consideradas. Os componentes principais são frequentemente usados como entrada para outras análises, como a regressão. Se os PC's são usados como variáveis preditoras, o modelo de regressão é chamado de regressão do PCA. Como os PC's são construídos como combinações lineares de todas as variáveis, o número de PC's é igual ao número de variáveis preliminares. O critério de autovalor mostra o grau de significância de cada um dos PC's [7]. Esses PC's são inseridos no modelo de regressão, conforme Equação 2.

$$
\begin{aligned}
& \mathrm{Y}=\mathrm{B}_{1} \times \mathrm{PC}_{1}+\mathrm{B}_{2} \times \mathrm{PC}_{2}+\mathrm{B}_{3} \times \mathrm{PC}_{3}+\mathrm{B}_{4} \times \mathrm{PC}_{4}+ \\
& \mathrm{B}_{5} \times \mathrm{PC}_{5}+\mathrm{B}_{6} \times \mathrm{PC}_{6}+\mathrm{B}_{7} \times \mathrm{PC}_{7}+\mathrm{B}_{0}
\end{aligned}
$$

Os parâmetros obtidos pela regressão do PCA não são válidos se aplicarmos diretamente a equação aos dados originais, sendo necessário neste caso retornar as variáveis aos seus estados iniciais. Assim, os parâmetros obtidos (coeficientes $B_{i}$ como um vetor, sem o $B_{0}$ ) são multiplicados pelos autovetores da matriz de correlação para calcular os coeficientes do modelo final, que pode então ser utilizado pelos dados sem tratamento [8]. Cada elemento nos autovetores mostra a parcela do parâmetro preliminar específico de cada PC [9]. Se a multiplicação do vetor $B$ com a matriz transposta de autovetores for denominada vetor $A$, as funções de custo final podem ser obtidas da Equação 3.

$$
\begin{aligned}
& \mathrm{Y}=\mathrm{A}_{1} \times\left(\frac{\mathrm{T}-\mathrm{T}_{\text {ave }}}{\mathrm{S}_{\mathrm{T}}}\right)+\mathrm{A}_{2} \times\left(\frac{\mathrm{C}-\mathrm{C}_{\text {ave }}}{\mathrm{S}_{\mathrm{C}}}\right)+\mathrm{A}_{3} \times\left(\frac{\mathrm{S}-\mathrm{S}_{\text {ave }}}{\mathrm{S}_{\mathrm{S}}}\right)+\mathrm{A}_{4} \times\left(\frac{\mathrm{G}-\mathrm{G}_{\text {ave }}}{\mathrm{S}_{\mathrm{G}}}\right)+ \\
& \mathrm{A}_{5} \times\left(\frac{\mathrm{K}-\mathrm{K}_{\text {ave }}}{\mathrm{S}_{\mathrm{K}}}\right)+\mathrm{A}_{6} \times\left(\frac{\mathrm{H}-\mathrm{H}_{\text {ave }}}{\mathrm{S}_{\mathrm{H}}}\right)+\mathrm{A}_{7} \times\left(\frac{\mathrm{V}-\mathrm{V}_{\text {ave }}}{\mathrm{S}_{\mathrm{V}}}\right)+\mathrm{A}_{0}
\end{aligned}
$$

Onde $T_{a v e^{\prime}} C_{a v e^{\prime}}, S_{a v e^{\prime}} G_{a v v^{\prime}}, K_{a v e^{\prime}}, H_{a v e}$ e $V_{a v e}$ são os valores médios, $S_{T}, S_{C}, S_{S}, S_{G}, S_{K}, S_{H}$ e $S_{V}$ são os valores de desvio padrão das variáveis porte do caminhão $(T)$, capacidade $(C)$, top size $(S)$, retido em $100 \mathrm{~mm}(G)$, limite de material compacto $(K)$, altura do prédio $(H)$ e volume da moega $(V)$, obtidas da Tabela 1. A Equação 3 pode ser diretamente manipulada utilizando as variáveis técnicas acima selecionadas.

\subsection{Equações de O'Hara}

Em análise complementar foi realizado outro teste com os dados reais disponíveis. Após levantamento dos principais modelos paramétricos existentes foi escolhido utilizar as equações originais de O'Hara de 1980 [2] e as equações atualizadas de 1992 [3]. Autores como Dehkharghani [10] se propuseram a fazer uma atualização monetária e cambial das equações de O'Hara [3], extrapolando estas equações para outras regiões do mundo. Nesta pesquisa ao invés de propôr uma atualização monetária e cambial das equações 
Tabela 2. Matriz de correlação

\begin{tabular}{|c|c|c|c|c|c|c|c|}
\hline Variáveis & $\mathrm{C}$ & $\mathbf{V}$ & $T$ & $\mathbf{S}$ & G & $\mathbf{K}$ & $\mathbf{H}$ \\
\hline $\mathrm{C}$ & 1 & 0,803 & $\mathbf{0 , 7 2 7}$ & 0,889 & $-0,071$ & $-0,364$ & 0,686 \\
\hline V & 0,803 & 1 & 0,873 & 0,770 & $-0,172$ & $-0,485$ & 0,793 \\
\hline $\mathrm{T}$ & 0,727 & 0,873 & 1 & 0,617 & $-0,180$ & $-0,481$ & 0,565 \\
\hline $\mathrm{S}$ & $\mathbf{0 , 8 8 9}$ & $\mathbf{0 , 7 7 0}$ & 0,617 & 1 & 0,317 & $-0,117$ & 0,657 \\
\hline G & $-0,071$ & $-0,172$ & $-0,180$ & 0,317 & 1 & 0,682 & $-0,095$ \\
\hline K & $-0,364$ & $-0,485$ & $-0,481$ & $-0,117$ & 0,682 & 1 & $-0,449$ \\
\hline $\mathrm{H}$ & 0,686 & 0,793 & 0,565 & 0,657 & $-0,095$ & $-0,449$ & 1 \\
\hline
\end{tabular}

Tabela 3. Vetor B calculado para os Componentes Principais selecionados

\begin{tabular}{cccc}
\hline & Coeficiente B & Desvio padrão & Valor-P \\
\hline Interseção $\mathrm{B}_{0}$ & 42,406 & 1,45 & 0,000 \\
$\mathrm{~B}_{1}$ para $\mathrm{PC}_{1}$ & 6,542 & 0,71 & 0,000 \\
$\mathrm{~B}_{2}$ para $\mathrm{PC}_{2}$ & 2,971 & 1,11 & 0,018 \\
$\mathrm{~B}_{3}$ para $\mathrm{PC}_{3}$ & 0,841 & 2,18 & 0,706 \\
$\mathrm{~B}_{4}$ para $\mathrm{PC}_{4}$ & $-4,357$ & 2,61 & 0,117 \\
$\mathrm{~B}_{5}$ para $\mathrm{PC}_{5}$ & 9,349 & 2,97 & 0,007 \\
$\mathrm{~B}_{6}$ para $\mathrm{PC}_{6}$ & $-2,395$ & 5,07 & 0,644 \\
$\mathrm{~B}_{7}$ para $\mathrm{PC}_{7}$ & 35,622 & 19,85 & 0,094 \\
\hline
\end{tabular}

de O'Hara, o que se mostrou extremamente complexo e de baixa eficácia em função da significativa disparidade de data-base, de realidade de poder de compra e de custos de fornecimentos e serviços entre Brasil e Estados Unidos; foi proposto aplicar um fator multiplicador de ajuste nestas equações. Utilizando a base de dados reais disponível, a proposta foi obter o menor erro médio possível comparando as equações de O'Hara [3] com estes dados reais, utilizando ferramenta de otimização do Microsoft Excel (Solver), de forma a determinar o fator de ajuste ótimo gerador do erro médio mínimo, quando comparados os dados reais e calculados. Desta forma, foi possível calibrar as equações originais (1980) [2] e atualizadas (1992) de O'Hara [3], trazendo-as da base de custos norte americana desatualizada para a base de custos atualizada da mineração brasileira.

Equação original de 1978 para estimativa macro de custos de instalações de britagens primária e secundária (Equação 4):

$$
\mathrm{Y}=\$ 67050 X^{0,5}
$$

Onde $X$ se refere à tonelagem britada por dia e $Y$ o custo de investimento em milhões de dólares.Equações atualizadas de 1988 para custos de britadores giratórios, planta de britagem primária e planta de britagem de finos respectivamente apresentadas pelas Equações 5, 6 e 7:

$$
\begin{aligned}
& Y=\$ 63 X^{0,9} \\
& Y=\$ 15000 X^{0,7} \\
& Y=\$ 18000 X^{0,7}
\end{aligned}
$$

\section{Resultados e discussão}

\subsection{Análise de componentes principais}

Após análise estatística dos dados foi identificado que as variáveis consideradas possuem alta correlação entre si, o que justifica a aplicação da análise de componentes principais neste caso.

A Tabela 2 mostra a matriz de correlação das variáveis para os dados das britagens primárias e secundárias levantadas, com valores acima de 0,7 destacados (alta correlação).

Tais correlações foram omitidas usando o método PCA e foram construídos novos modelos de regressão baseados nos novos PC's, resultando na introdução dos coeficientes de modelo $\left(B_{i}\right.$ 's), como mostrado na Tabela 3.

Como esses coeficientes não são capazes de identificar as relações diretas entre os custos e as variáveis, faz-se necessário transformar o modelo (e subsequentemente os coeficientes) em uma nova situação inicial. Os autovetores introduzidos na Seção 2.2 foram utilizados para permitir a concretização deste objetivo. A Tabela 4 mostra os autovetores da matriz de correlação das variáveis das britagens.

A análise de componentes principais permite avaliar a possibilidade de reduzir o conjunto de variáveis, através dos autovalores ou valores característicos (Eigenvalues). Estes valores devem ser maiores do que 1 para os PC's selecionados, conforme critério de Kaiser [7].

Considerando os valores superiores a 1 na Figura 3, percebe-se que os componentes principais $F_{1}$ (ou $P C_{1}$ ) e $F_{2}\left(\right.$ ou $\left.P C_{2}\right)$ descrevem aproximadamente $85 \%$ da variância do conjunto total, o que é representativo. Ou seja, a análise de componentes principais suporta a decisão de se reduzir o número de variáveis originais.

Desta forma, neste artigo foi avaliado trabalhar com dois componentes principais suportado pela análise mostrada na Figura 3. Para efeito de comparação, também foram avaliados os resultados e erros associados mantendo-se os sete componentes principais originais. Por sua vez, através dos factor loadings, pode-se afirmar que as variáveis $C, V, T, S, e$ $H$ estão muito bem correlacionadas ao fator 1 (componente principal 1) e as variáveis $G$ e $K$ ao fator 2 (componente principal 2). Está indicado em destaque na Tabela 5 os coeficientes de correlação acima de 0,7 (valores em negrito). A Figura 4 mostra graficamente esta correlação. 
Tabela 4. Autovetores (Eigenvectors)

\begin{tabular}{cccrrrrrr}
\hline Variáveis & F1 & F2 & F3 & F4 & F5 & F6 & F7 \\
\hline C & 0,443 & 0,136 & $-0,092$ & $-0,634$ & 0,057 & $-0,391$ & $-0,468$ \\
V & 0,467 & 0,009 & $-0,097$ & 0,292 & 0,230 & 0,656 & $-0,451$ \\
T & 0,421 & $-0,042$ & $-0,625$ & 0,442 & $-0,003$ & $-0,412$ & 0,254 \\
S & 0,400 & 0,398 & 0,018 & $-0,345$ & $-0,172$ & 0,362 & 0,634 \\
G & $-0,089$ & 0,713 & 0,065 & 0,320 & $-0,525$ & $-0,103$ & $-0,303$ \\
K & $-0,274$ & 0,559 & $-0,156$ & 0,005 & 0,761 & $-0,058$ & 0,069 \\
H & 0,407 & 0,027 & 0,750 & 0,310 & 0,243 & $-0,319$ & 0,121 \\
\hline
\end{tabular}

Tabela 5. Factor loadings

\begin{tabular}{|c|c|c|c|c|c|c|c|}
\hline Variáveis & F1 & F2 & F3 & F4 & F5 & F6 & F7 \\
\hline $\mathrm{C}$ & 0,909 & 0,177 & $-0,062$ & $-0,353$ & 0,028 & $-0,112$ & $-0,034$ \\
\hline V & 0,959 & 0,012 & $-0,064$ & 0,162 & 0,113 & 0,188 & $-0,033$ \\
\hline $\mathrm{T}$ & 0,865 & $-0,055$ & $-0,416$ & 0,246 & $-0,002$ & $-0,118$ & 0,019 \\
\hline S & 0,820 & 0,520 & 0,012 & $-0,192$ & $-0,084$ & 0,104 & 0,046 \\
\hline G & $-0,183$ & 0,930 & 0,044 & 0,178 & $-0,257$ & $-0,029$ & $-0,022$ \\
\hline K & $-0,563$ & 0,730 & $-0,104$ & 0,003 & 0,373 & $-0,017$ & 0,005 \\
\hline $\mathrm{H}$ & 0,834 & 0,036 & 0,500 & 0,173 & 0,119 & $-0,091$ & 0,009 \\
\hline
\end{tabular}

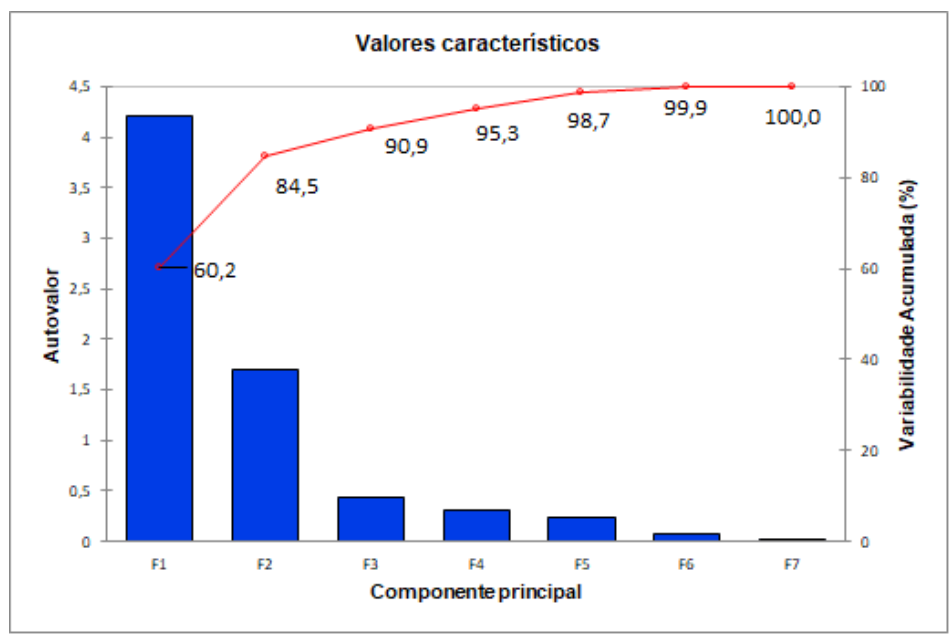

Figura 3. Valores característicos.

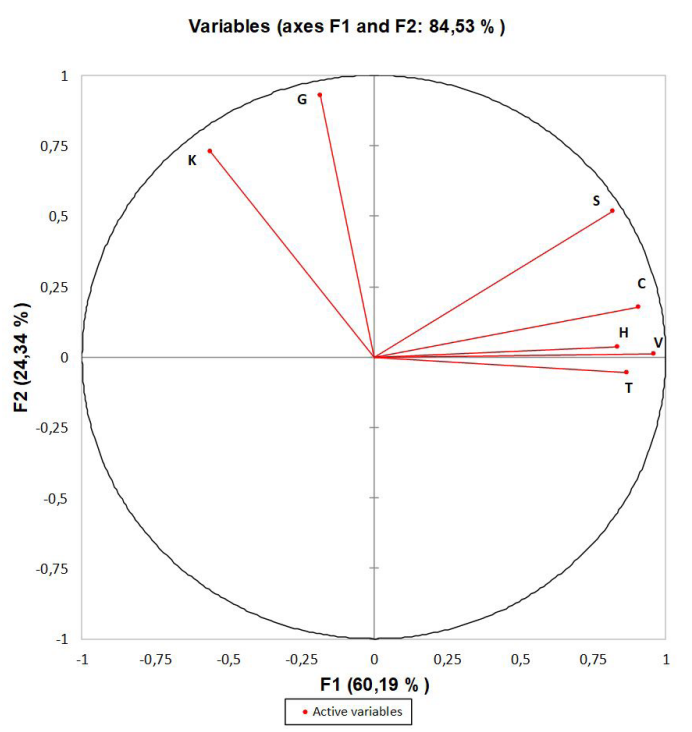

Figura 4. Coeficientes de correlação das variáveis com os fatores 1 e 2 .
Outra análise de destaque passa por avaliar quais observações (denominados itens na Tabela 1) estão contribuindo de forma mais relevante para cada fator. Nesta análise, mostrada na Tabela 6 , fica claro que para o fator 1 existem vários itens de contribuição, sem destaque para um item único, e para o fator 2 existe maior concentração entre os itens, já que somente o item 7 contribui com $56 \%$ deste fator. Os maiores valores estão destacados em negrito na Tabela 6.

Os factor loadings, como mostrado anteriormente na análise da Tabela 6 , foram úteis para avaliar quais fatores estão descrevendo quais variáveis. Já para identificação de grupos ou clusters utilizou-se a pontuação dos fatores, denominados scores. Os coeficientes dos modelos finais foram calculados pela aplicação das Equações 2 e 3, considerando apenas dois PC's e também todos os sete PC's originais. A Tabela 7 apresenta os coeficientes dos modelos de regressão final $\left(A_{1}, A_{2}, A_{3}, A_{4}, A_{5}, A_{6} e A_{7}\right)$ e o novo intercepto $\left(A_{0}\right)$. 
Tabela 6. \% de contribuição dos itens para cada fator

\begin{tabular}{|c|c|c|c|c|c|c|c|}
\hline Item & F1 & F2 & F3 & F4 & F5 & F6 & F7 \\
\hline 1 & 5,415 & 0,608 & 1,237 & 2,289 & 1,942 & 0,868 & 1,410 \\
\hline 2 & 5,415 & 0,608 & 1,237 & 2,289 & 1,942 & 0,868 & 1,410 \\
\hline 3 & 0,129 & 2,312 & 17,873 & 22,083 & 0,864 & 5,584 & 0,144 \\
\hline 4 & 0,429 & 2,218 & 6,479 & 30,578 & 2,785 & 0,492 & 4,366 \\
\hline 5 & 5,465 & 0,040 & 24,877 & 15,915 & 6,245 & 0,663 & 0,264 \\
\hline 6 & 0,500 & 6,687 & 0,104 & 0,010 & 4,229 & 10,658 & 1,348 \\
\hline 7 & 0,853 & 56,763 & 0,000 & 10,370 & 25,155 & 1,924 & 0,345 \\
\hline 8 & 4,065 & 0,660 & 0,428 & 0,411 & 4,708 & 0,661 & 1,974 \\
\hline 9 & 4,065 & 0,660 & 0,428 & 0,411 & 4,708 & 0,661 & 1,974 \\
\hline 10 & 8,576 & 0,349 & 2,336 & 0,091 & 0,670 & 1,179 & 0,729 \\
\hline 11 & 11,076 & 0,400 & 14,437 & 0,671 & 3,301 & 1,340 & 6,146 \\
\hline 12 & 9,194 & 0,362 & 4,483 & 0,000 & 1,161 & 0,254 & 0,000 \\
\hline 13 & 4,748 & 0,632 & 0,073 & 1,205 & 3,109 & 0,010 & 0,002 \\
\hline 14 & 4,748 & 0,632 & 0,073 & 1,205 & 3,109 & 0,010 & 0,002 \\
\hline 15 & 4,431 & 0,645 & 0,022 & 0,792 & 3,795 & 0,099 & 0,441 \\
\hline 16 & 0,715 & 7,036 & 0,169 & 0,398 & 4,545 & 5,714 & 8,650 \\
\hline 17 & 0,479 & 6,679 & 0,165 & 0,003 & 4,079 & 11,207 & 1,077 \\
\hline 18 & 0,029 & 5,764 & 3,037 & 1,941 & 0,040 & 4,473 & 37,486 \\
\hline 19 & 6,306 & 6,256 & 12,974 & 0,099 & 20,636 & 40,308 & 4,061 \\
\hline 20 & 8,971 & 0,357 & 3,636 & 0,013 & 0,970 & 0,507 & 0,089 \\
\hline 21 & 6,737 & 0,309 & 0,133 & 1,528 & 0,000 & 8,734 & 13,189 \\
\hline 22 & 7,655 & 0,024 & 5,798 & 7,698 & 2,010 & 3,785 & 14,891 \\
\hline
\end{tabular}

Tabela 7. Coeficientes dos modelos de regressão do PCA testados

\begin{tabular}{cccccrrrr}
\hline $\begin{array}{c}\text { Componentes } \\
\text { selecionados }\end{array}$ & $\mathbf{A}_{\mathbf{0}}$ & $\mathbf{A}_{\mathbf{1}}$ & $\mathbf{A}_{\mathbf{2}}$ & $\mathbf{A}_{\mathbf{3}}$ & $\mathbf{A}_{\mathbf{4}}$ & $\mathbf{A}_{\mathbf{5}}$ & $\mathbf{A}_{\mathbf{6}}$ & $\mathbf{A}_{\mathbf{7}}$ \\
\hline $\mathrm{PC}_{1}$ e $\mathrm{PC}_{2}$ & 42,406 & 3,299 & 3,085 & 2,633 & 3,797 & 1,533 & $-0,132$ \\
$\mathrm{PC}_{1}$ a PC & 42,406 & $-9,220$ & $-13,74$ & 10,191 & 25,433 & $-15,247$ & 9,415 & 9,741 \\
\hline
\end{tabular}

A fim de se verificar o ajuste dos modelos calculou-se o erro conforme Equação 1 dos modelos de regressão calculados. Além dos modelos de regressão do PCA, para comparação foi calculado o erro para a regressão multivariada utilizando método stepwise (passo-a-passo), sem omissão de correlação entre as variáveis (Tabela 8). O método stepwise utiliza a seleção forward e backward, em que é avaliada através de estatísticas (P, F-parcial) a significância de cada variável ao modelo. As variáveis descartadas na regressão stepwise foram as variáveis $C, V \mathrm{e} T$, pois apresentaram valores de $\mathrm{P}$ superiores a $5 \%$ (intervalos de confiança inferiores a 95\%).

\subsection{Ajuste das equações de O’Hara}

Seguem resultados na Tabela 9 dos fatores multiplicadores de ajuste ótimos a serem aplicados sobre as equações originais e atualizadas de O'Hara [2,3].

No teste realizado comparando a equação original com os dados reais, em 16 dos 22 dados comparados (73\%) o erro foi inferior a $30 \%$. No teste realizado comparando as equações atualizadas em 18 dos 22 casos (82\%) o errou foi inferior a $30 \%$. Ou seja, o fator de ajuste aplicado às equações foi satisfatório, mostrando que estas equações podem ser utilizadas com bom nível de assertividade em estudos de pré-viabilidade e viabilidade.
Tabela 8. Erro médio relativo

\begin{tabular}{cc}
\hline Modelo de regressão & Erro (\%) \\
\hline PCA (2 PC's) & $17,7 \%$ \\
PCA (7 PC's) & $\mathbf{9 , 7 \%}$ \\
Stepwise & $10,9 \%$ \\
\hline
\end{tabular}

Tabela 9. Fatores de ajuste

\begin{tabular}{ccc}
\hline Equação O'Hara & Fator de ajuste & Erro médio (\%) \\
\hline Original & 1,661 & $20 \%$ \\
Atualizada & 0,620 & $17 \%$ \\
\hline
\end{tabular}

\section{Conclusão}

O modelo univariado é apropriado para estimativas preliminares de custos quando não existem informações suficientes disponíveis. Entretanto, com a incorporação de outras variáveis relevantes ao modelo, ou seja, tornando-o multivariado, pode-se agregar valor e assertividade às estimativas. A omissão da correlação entre os parâmetros foi realizada pelo método PCA. Os erros calculados indicam que o modelo multivariado pode ser usado como uma ferramenta poderosa para a estimativa de custos de instalações de britagens primárias e secundárias. O modelo de melhor ajuste foi o modelo de regressão do PCA utilizando todos os 
componentes principais. Este modelo apresentou erro médio inferior a $10 \%$, mostrando-se adequado para as estimativas de investimentos aplicadas a estudos de pré-viabilidade e de viabilidade. Como a quantidade de componentes principais escolhidos afeta o quanto os dados podem ser explicados pelos componentes (como mostrado na Figura 3), é plausível que o erro apresentado utilizando apenas dois componentes principais fosse superior a $15 \%$. Dessa forma, a quantidade de componentes pode ser escolhida de acordo com o quanto os dados são explicados, ao invés de se utilizar o critério de Kaiser [7]. Ou seja, o percentual de explicação dos dados pode ser utilizado como critério para a redução de componentes, mas como no caso estudado a quantidade de componentes é relativamente reduzida, todos os componentes podem ser mantidos na análise.

Com relação aos testes com as equações de O'Hara [3] os resultados encontrados foram satisfatórios, mostrando boa aproximação com relação aos dados reais coletados. Isto comprova que as equações de O'Hara [3] seguem sendo úteis para estimativas preliminares de investimento, desde que seja aplicado o fator de ajuste recomendado sobre estas equações. Recomenda-se que a mesma metodologia seja utilizada para outras operações unitárias de mineração (como por exemplo prédios de peneiramento, moagem, flotação, entre outros).

\section{Referências}

1 Sayadi AR, Khalesi MR, Borji MK. A parametric cost model for mineral grinding mills. Minerals Engineering. 2014;55:96-102.

2 O'Hara TA. Quick guide to the evaluation of ore bodies. CIM Bulletin. 1980;88:34-43.

3 O'Hara TA, Suboleski CS. Costs and cost estimation. In: O'Hara TA. SME mining engineering handbook. USA: Society for Mining, Metallurgy, and Exploration; 1992. p. 405-424. Chap. 6.3.

4 Prasad L. Mineral processing plant design and cost estimates. Montreal: Processors Division of Canadian Institute of Mining, Metallurgy and Petroleum; 1969.

5 Mular AL. Mining and mineral processing equipment costs and preliminary capital cost estimations. Montreal: Canadian Mining and Metallurgy Institue; 1982.

$6 \mathrm{Kim}$ GH, An SH, Kang KI. Comparison of construction cost estimating models based on regression analysis, neural networks and case-based reasoning. Building and Environment. 2004;39(10):1235-1242.

7 Kaiser HF. The application of electronic computers to factor analysis. Educational and Psychological Measurement. 1960;20:141-151.

8 Timm, N.H. Applied Multivariate Analysis. New York: Springer-Verlag; 2002.

9 Sayadi AR, Lashgari A, Paraszczak J. Hard-rock LHD cost estimation using single and multiple regression based on principle component analysis. Tunnelling and Underground Space Technology. 2012;27(1):133-141.

10 Dekharghani AA, Osanloo M. An updated and modified O'Hara cost estimating model based on World and Iran economic conditions. In Dessureault S, Ganguli R, Kecojevic V, Girard-Dwyer J, editors. Application of computers and operations research in the mineral industry. London: Taylor \& Francis Group; 2005.

Recebido em: Jan. 31, 2020

Aceito em: Out. 19, 2020. 